\title{
Zur Überführung von Ergebnissen der neueren Polymer- forschung in die Technologie der Kunststoffverarbeitung
}

\author{
Prof. Dr. rer: nat. habil. Rolf Hirte, Dipl.-Phys. Jörg-Peter Melior
}

\section{Zusammenfassung}

Die erwünschte Nutzung von Ergebnissen der Grundlagenforschung in der Verarbeitungspraxis der Kunststoffe setzt zunächst voraus, daß geeignete Charakterisierungsmöglichkeiten für die in Verarbeitungsprozessen ablaufenden Strukturbildungsprozesse geschaffen werden. Erst die Verfügbarkeit solcher Methoden ermöglicht die gezielte Einflußnahme auf Strukturbildungsvorgänge in der Produktionspraxis. Die Erprobung solcher Methoden innerhalb der studentischen Praktika bietet eine motivierende Herausforderung und sensibilisiert fuir die Probleme der wissenschaftlich fundierten Optimierung von technologischen Prozessen.

\section{Einleitung}

Der ökologische Wandel unserer Volkswirtschaft zur „nachhaltigen“ Wirtschaft verlangt höhere Energie- und Ressourceneffizienz. Die konkreten Anforderungen, die in dieser Hinsicht an moderne Werkstoffe zu stellen sind, werden vielfach von Kunststoffen am besten erfuillt, weil ihre Herstellung, Verarbeitung und ihr Recycling relativ ressourcenschonend, weitgehend abproduktfrei und mit geringerem Energieaufwand gestaltet werden kann, als es bei konkurrierenden metallischen oder mineralischen Werkstoffen möglich ist. Kunststoffe besitzen daruiber hinaus spezifische Vorteile wie geringes spezifisches Gewicht, hohe Variabilität der Anwendungseigenschaften, beste Isolier- und Trenneigenschaften, höchste chemische und biologische Beständigkeit.

Das gegenwärtige Einsatzspektrum von Kunststoffen ist stark von der an sich positiven Tatsache bestimmt, daß Kunststoffprodukte sehr billig sind. Es gibt zwar Einsatzfälle, in denen Kunststoffe mit ihren besonderen Eigenschaften gefordert und als unverzichtbar anerkannt sind. Daneben gibt es aber auch viele kritikwürdige Einsatzfälle, in denen Kunststoffe als bloße Ersatzstoffe mißbraucht werden. Die Zukunft des Kunststoffeinsatzes liegt in höherwertigem Einsatz, in stärkerer Ausnutzung polymerspezifischer Materialeigenschaften, welche durch die Anisotropie der Makromolekuile ermöglicht und durch Manipulation der Strukturausbildung variierbar sind.

\section{Über Möglichkeiten der durchgreifenden Verbesserung von Eigenschaften spezieller Kunststoffprodukte}

Die werkstofftechnischen Besonderheiten der Kunststoffe beruhen auf der Kettenstruktur der Polymermolekiile. Als Keller (Bristol) in den fuinfziger Jahren entdeckte, daß Polymermoleküle völlig unerwartet in gefalteter Form kristallisieren, begann man intensiv, die Frage des Zusammenhanges von Struktur und Eigenschaften von polymeren Werkstoffen zu untersuchen. Wenn die Polymerketten ihre Längsausdehnung gleichsam verschenken, d. h. wenn die Kettennatur der Polymermoleküle im werkstofflichen Einsatz nicht genutzt wird, wovon werden die Eigenschaften polymerer Werkstoffe dann bestimmt und wie lassen sie sich beeinflußen? Die Bemuihungen der Polymerphysiker um Aufklärung zunächst der Feinstruktur von Polymeren und später der Strukturbildung waren sehr erfolgreich. Auch für den Praxiserfolg solcher Bemuihungen gibt es zwar nur wenige, dafuir aber überzeugende Beispiele. Speziell sind hier die in der folgenden Tabelle enthaltenen fadenförmigen Hochfestmaterialien, C-Faser und Kevlar zu nennen, deren Verfügbarkeit eine Revolution im Leichtbau ausgelöst hat.

Die Kettenfaltung ließ sich durch Versteifung des Polymermoleküls unterdruicken. Diese Erkenntnis führte zur Entwicklung von Spezialpolymeren, die ohne großen Aufwand zur gestrecktkettigen Kristallisation veranlaßt werden können. Das bekannteste Produkt ist der hochfeste Kevlar-Faden. Mit der Entdeckung der Möglichkeit der gestrecktkettigen Kristallisation auch von flexibelkettigen Polymeren durch Wunderlich [3] und der gezielten Herstellung solcher Materialien durch Pennings [15] in gescherten Lösungen wurde der Prototyp von hochfestem Polymermaterial mit flexiblen Ketten erzeugt und untersucht. Als „Gelfaden“ ist ein hochfestes Polyethylen dieser speziellen Struktur inzwischen auch kommerziell verfuigbar.

Um zu illustrieren, welche Eigenschaftsreserven in polymeren Werkstoffen stecken, ist in Tabelle 1 für den speziellen Fall linearer Festigkeitsträger, wie sie zur Verstärkung in Kunststoff- oder Elastomerverbunden verwendet werden, ein Eigenschaftsvergleich angegeben. 


\begin{tabular}{|l|c|c|c|c|}
\hline & $\begin{array}{c}\text { Dichte } \\
\text { in g/cm }\end{array}$ & $\begin{array}{c}\text { Zugfestigkeit } \\
\text { in GPa }\end{array}$ & $\begin{array}{c}\text { E-Modul } \\
\text { in GPa }\end{array}$ & $\begin{array}{c}\text { Restdehnung } \\
\text { in \% }\end{array}$ \\
\hline $\begin{array}{l}\text { Kohlenstoff- } \\
\text { Faser }\end{array}$ & 1,78 & 3,1 & bis 250 & $0,9-1,2$ \\
Glasfaser, S-Glas & 2,54 & 3,3 & 88 & $3-4$ \\
Stahlcord & 7,83 & 3,1 & 210 & $2-3$ \\
$\begin{array}{l}\text { Nylon- } \\
\text { Reifencord }\end{array}$ & 1,13 & 1 & 6 & 18 \\
$\begin{array}{l}\text { Polyester- } \\
\text { Reifencord }\end{array}$ & 1,23 & 1,1 & 14 & 15 \\
Kevlar & 1,44 & 2,8 & 124 & 2,8 \\
$\begin{array}{l}\text { Polyethylen, } \\
\text { lösungsgesponnen }\end{array}$ & 0,97 & $\sim 3$ & $\sim 30$ & $<5$ \\
$\begin{array}{l}\text { Polyethylen, } \\
\text { lösungsgespon- }\end{array}$ & & & & \\
nen, gereckt & $\sim 1$ & bis 9,8 & bis 130 & $<2$ \\
\hline
\end{tabular}

Tabelle 1: Mechanische Kennwerte ausgewählter Hochfestmaterialien; aus der Literatur zusammengestellt, Polyethylen [16]

Die spezifischen Eigenschaften von Polymerketten werden bei der Herstellung von C-Faser, bei dem steifkettigen Polymermaterial Kevlar sowie den lösungsgesponnenen Fäden genutzt, während die übrigen Materialien nach klassischen Technologien hergestellt werden. Dabei handelt es sich im Falle der C-Faser und des Kevlar um teure Spezialentwicklungen, die den hohen Materialpreis bedingen. Die Polyethylenfäden dagegen bestehen aus billigem, handelsuiblichem Material. Der Hinweis, daß für den Einsatz von Verstärkungsmaterialien weitere Anforderungen bestehen, bei denen die lösungsgesponnenen Fäden nicht immer so deutlich den Vorzug verdienen, darf hier aber nicht fehlen.

Wenn die mikrophysikalischen Eigenschaften der Polymerkette für den makroskopischen Körper nutzbar gemacht werden, dann wird eine Verbesserung des allgemeinen Eigenschaftsniveaus ermöglicht. Soweit man die verbesserten mechanischen Eigenschaften nutzen will, ermöglichen sie in vielen Einsatzfällen eine erhebliche Verschlankung von Bauteilen, also Materialeinsparung. Hochgeordnete Polymere besitzen aber nicht nur bessere mechanische Eigenschaften, sie werden auch widerstandsfähiger gegen chemischen und biologischen Angriff, ihre elektrischen und optischen Eigenschaften verändern sich, ihre thermische Belastbarkeit nimmt zu. Es eröffnen sich für solche Materialien völlig neue Einsatzgebiete. Wenn es gelingt, für eingefuihrte Massenpolymere die gewünschten Eigenschaften mittels veränderter, nur unwesentlich aufwendigerer Verarbeitungstechnologien zu verbessern, entstehen keine neuen Recyclingprobleme und man kann mit guten Gründen von höherwertigem Kunststoffeinsatz sprechen.

Diese wünschenswerten Verbesserungen setzen aber ein gruindliches Verständnis der übermolekularen Strukturen und der polymerspezifischen Strukturbildungsgesetzmäßigkeiten voraus. Die wissenschaftlichen Bemühungen um die Aufklärung dieser Zusammenhänge klärten nicht nur die theoretischen Zusammenhänge und die Charakterisierungsprobleme sondern fuihrten auch zur Entwicklung einer Reihe von Labortechnologien, welche diese Zusammenhänge nutzen, um gestrecktkettig kristallisiertes Material zu erzeugen. Solche Verfahren sind die Festkörperextrusion, Ultra-Reckung, Hochdruckkristallisation, Orientierungskristallisation, Gelspinnen. Eine Übersicht ist zu finden in [5]. Die wichtigste Besonderheit soll noch einmal hervorgehoben werden: Es handelt sich um flexibelkettige Polymere, zu denen insbesondere auch alle billigen Massenkunststoffe gehören. Die Ergebnisse hätten allerhöchste Praxisrelevanz, wenn es gelänge, diesen Forschungsstand in der Kunststoffverarbeitung wirksam werden zu lassen.

Die direkte Überführung theoretischer Kenntnisse der Strukturbildung in die alltägliche Praxis der Kunststoffverarbeitung stellt ein großes Problem dar. Die Technologie der Kunststoffverarbeitung, speziell der Massenkunststoffe, ist in Bezug auf niedrige Stiickkosten und gleichbleibendes Qualitätsniveau weitgehend ausoptimiert. Gut gemeinte Veränderungen am technologischen Regime von Produktionsanlagen führen mit größter Wahrscheinlichkeit zu katastrophalen Einbrüchen bei Kosten und/oder Qualität. Trotzdem ist die Bemühung um stärkere Beriicksichtigung polymerspezifischer „Neigungen“ der Strukturbildung auch unter den Bedingungen der herkömmlichen Verarbeitungstechnik nicht sinnlos. Untersuchungen zur „Eigenverstärkung“ von flexibelkettigen, kristallisierenden Polymeren wie Polyethylen, Polypropylen u. A. beim Spritzgießen über die z. B. Ehrenstein und Mitarbeiter in [8] berichten, wiesen in diese Richtung und waren sehr erfolgreich.

\section{Problem der Überführung relevanter Forschungsergebnisse in die Kunststoff- verarbeitung}

Der geschilderte Stand der polymerphysikalischen Forschung legt die Frage nahe, warum noch immer keine Revolution der Verarbeitungstechnologien stattgefunden hat und die Kunststoffverarbeitung von diesen Ergebnissen scheinbar unbeeindruckt bleibt. Der Grund ist einfach: Der Kenntnisstand über die Strukturbildung innerhalb üblicher Verarbeitungsprozessen ist nach wie vor zu lückenhaft, um beispielsweise die Möglichkeiten, die die Eigenverstärkung bietet, routinemäßig bei allen Spritzgießprozessen nutzen zu können, denn unter Verarbeitungsbedingungen können Strukturbildungsvorgänge nur in seltenen Ausnahmefällen messend verfolgt werden. Der naheliegende Ausweg, die Prozesse im Labor zu modellieren und sie auf diese Weise der Messung zugänglich zu machen, scheidet weitgehend aus, weil die Nachbildung des komplexen Zusammenspiels von Temperatur und Druck, von Scherung, Relaxation und Erstarrung, wie sie im Verarbeitungsprozeß ablaufen, einen viel zu hohen Aufwand erfordern würde. Kenntnis der strukturellen Abläufe bildet aber die Voraussetzung aller Optimierungsbemühungen.

Der Ausweg, den wir für gangbar halten, versucht einen Kompromiß: Ausgehend von beherrschten Zusammenhängen, durch schrittweise Annäherung an die Praxisbedingungen wenigstens die Tendenz der Zusammenhänge aufzuklären. Das ermöglicht es, später mit Mitteln der gezielten Technologievariation die Auswirkung auf das Produkt auszudehnen.

Konkret gilt unser Interesse der Kristallisation unter 
Deformation des flüssigen Polymermaterials („Scherkristallisation").

Die Untersuchung der Kristallisation unter Deformation stellt den Experimentator vor große Probleme. Orientierung der Ketten, Keimbildung und Kristallwachstum sind molekulare Prozesse, von denen die makroskopisch meßbaren Größen, wie Kristallinität oder Orientierung in sehr komplexer Weise abhängig sind. Eine starke Rückwirkung des bereits kristallisierten Materials auf die Strömungsverhältnisse führt zudem zu einer komplizierten Wechselbeziehung zwischen Orientierung und Kristallisation. Scherkristallisationsvorgänge können sich nicht bis zum Ende der Kristallisation fortsetzen. Entweder werden die Ergebnisse der Scherkristallisation durch sich anschließende Verstreckung modifiziert oder unter einem großen Anteil anschließend „normal“ kristallisierten Materials verborgen [7], [9], [13], [14]. Auch aus diesen Gründen ist es nicht möglich, innerhalb technologischer Prozesse eine ausreichende experimentelle Beobachtung durchzuführen.

Die beiden Extremfälle der Kristallisation (Ruhekristallisation, Kristallisation aus einem Zustand nahezu vollständiger Kettenstreckung heraus) und die daraus entstehenden uibermolekularen Strukturen, die in Faltlamellen organisierten Sphärolithe [11] einerseits, gestrecktkettig kristallisierte Fibrillen [3] andrerseits sind in der Vergangenheit sehr intensiv bezuiglich ihrer Struktur untersucht worden [1], [2], [4], [12]. Gesicherte Aussagen zur Kristallisationskinetik liegen aber nur für den Fall der Ruhekristallisation vor. Die Beschreibung des Kristallisationsprozesses sollte sich deshalb auch fuir kleine Scherung am Ruhekristallisationsfall orientieren. Für starke Deformation muß ein physikalisch begründetes Beschreibungsmodell, das auf Ergebnissen basiert, die bei kleinen Scherungen gewonnen wurden, erst erarbeitet werden.

Es ergibt sich eine mögliche Abfolge der vorgesehenen Bearbeitungsschritte:

1. Schaffung experimenteller und methodischer Voraussetzungen für Scherkristallisationsexperimente unter praxisnahen Bedingungen,

2. Untersuchung des Zusammenhangs von Scherung, Ausgangsorientierung und Kristallisationsgeschwindigkeit,

3. Erarbeitung und Testung praxisreifer Nutzungsvorschläge.

Für die unter 1 genannten Scherkristallisationsexperimente sind die apparativen Voraussetzungen geschaffen. In methodischer Hinsicht müssen Überlegungen und Testuntersuchungen angestellt werden, die den physikalischen Aussagegehalt der Messungen sichern. Dabei kann und muß man sich auf Meßgrößen zurïckziehen, die trotz erschwerter Bedingungen die erforderliche Aussagesicherheit bieten. Das bedeutet aber auch Verzicht auf Auswertungsmethoden, die sich im idealisierten Fall der Ruhekristallisation bewährt haben. Ein wichtiges Kriterium für die Methodenwahl ist die Minimierung des Meßaufwandes nicht aus forschungsökonomischen Gründen, sondern um den Zugriff auf den zu untersuchenden Prozeß zu verringern und damit Möglichkeiten der größeren Praxisnähe des Modell- prozesses zu eröffnen. Die Entwicklung und Testung solcher "Primitivmethoden" stellt einen interessante Herausforderung dar. Im Anhang soll beispielhaft eine solche methodische Vorüberlegung beschrieben werden.

\section{Anhang: Vereinfachte Auswertung kristalllisationskinetischer Messungen}

Für die messende Verfolgung von Kristallisationsprozessen stehen einen ganze Reihe von Methoden zur Verfügung. Ein wertender Überblick dazu wird an anderer Stelle gegeben werden. Im Blick auf eine Kristallisation unter verarbeitungnahen Bedingungen, ohne Beeinflussung durch den Meßvorgang, die zudem den zu messenden Geschwindigkeitsbereich abdecken kann, schränkt sich die Zahl der Methoden und der auswertbaren Größen stark ein. Es wird zu zeigen sein, daß bei Beachtung der theoretischen Zusammenhänge auch mittels einfachster Relativverfahren die erforderlichen physikalischen Aussagen zu gewinnen sind.

Bei der mikroskopischen Untersuchung von Kristallisationsprozessen in Polymeren mit dem Ziel der Aufklärung geschwindigkeitsverändernder Einflïsse wird gewöhnlich der Gesamtverlauf der Kristallisation z. B. durch unmittelbare Beobachtung von wachsenden Sphärolithen registriert. Man kann mit Vorteil aber auch viel einfacher zu bestimmende Induktionszeitmessungen benutzen. Diese Methode wird insbesondere in realen Systemen schnell kristallisierender Polymere notwendig, da die Registrierung der wachsenden Durchmesser von sehr kleinen morphologischen Einheiten im Polarisationsmikroskop nicht möglich ist. Hingegen ist die Bestimmung der Zeitdauer bis zum nachweisbaren Einsatz der Kristallisation mittels lichtempfindlicher Detektoren (Depolarisationsmessung) sehr einfach möglich. Hier soll der Zusammenhang dieser Induktionszeit mit kristallisationskinetischen Größen wie Keimbildungsrate oder linearer Kristallwachstumsgeschwindigkeit kurz beschrieben werden.

Beobachtet man den Beginn der isothermen Kristallisation, so setzt ein nachweisbarer Anstieg der Kristallinität erst nach einer Zeitspanne ein, die Induktionszeit genannt wird. In der Literatur wird der Begriff Induktionszeit oder auch Inkubationszeit im Zusammenhang mit der Kristallisation sehr unterschiedlich gedeutet.

1. Bei homogener bzw. thermischer Keimbildung ist nach Erreichen der Kristallisationstemperatur eine bestimmte Zeit nötig, bis sich eine stationäre Verteilung der Keimgrößen einstellt. Diese bildet die Voraussetzung fuir den zeitlich linearen Anstieg der Keimzahl d.h. eine konstante Keimbildungsrate I, gemäß der Formel von Turnbull und Fisher [10]:

$$
I=I_{0} \exp \left\{\frac{-\Delta G_{\eta}-\Delta \Phi}{k T}\right\}
$$

Wobei $\Delta \mathbf{G}_{\eta}$ die Aktivierungsenergie für den viskosen Fluß und $\Delta \Phi$ die Freie Energie der Keimbildung, $\mathrm{k}$ die Boltzmannkonstante und $\mathrm{T}$ die Temperatur be- 
deuten. Bei realen Kristallisationsprozessen ist der homogene Einsatz der Kristallisation aber nur in Ausnahmefällen, nämlich bei reinsten Substanzen und einer besonders gründlichen Schmelzebehandlung zu erreichen. Im Regelfall beginnt die Kristallisation heterogen, nämlich an Verunreinigungen, an unaufgeschmolzenen Kristallitresten oder in Bereichen verbliebener Vorordnung („Gedächtniseffekte“).

2. Bei sphärolithischer Kristallisation fuihrt die Extrapolation des Durchmessers heterogen gebildeter Sphärolithe auf Null nicht auch zum Nullpunkt der Zeit. Der so gewonnene Zeitpunkt kann bei negativen Zeitwerten liegen und fuihrte zur Bezeichnung ,negative Induktionszeit“. Die Ursache ist in dem Umstand zu suchen, daß Sphärolithe im Anfangsstadium ihrer Entwicklung garbenförmige Gebilde sind, die erst im Verlaufe des Wachstums durch Verzweigungen zu Kugeln werden. In diesem frühen Stadium gelten andere Wachstumsgesetze als bei den späteren kugelförmigen Sphärolithen. Die Auswirkung der notwendig zu durchlaufenden Wachstumsgeometrien auf den Verlauf der Bruttokristallisation ist sehr uniibersichtlich. Zwar kann man leicht mittels Modellrechnung den Verlauf der Grenzfälle rein homogener oder rein heterogener Keimbildung aufklären. Im Realfall ist der Bruttoeffekt aber von zu vielen Einflußgrößen abhängig. Die erwähnte Extrapolation muß mit Vorsicht vorgenommen werden. Es muß berücksichtigt werden, daß nur an Verunreinigungen, z. B. künstlichen Keimbildnern gestartete Sphärolithe sogleich als Kugeln wachsen und nur in diesem Falle der Kristallisationsprozess mit einheitlicher Wachstumsdimension beschrieben werden kann. Homogen gebildete Sphärolithkeime wachsen aber zunächst stäbchenförmig. Die notwendig zu durchlaufende Phase der Garbenform ist mathematisch mit Wachstumsdimensionen $>3 \mathrm{zu}$ beschreiben. Erst ausgewachsenen Sphärolithe wachsen wirklich dreidimensional. Wenn, wie z. B. bei PA6, der Hauptteil des Volumenzuwachses auf das garbenförmige Wachstum zurückzuführen ist, muß eine Extrapolation, die kugelförmiges Wachstum unterstellt, zu Fehlern fuihren.

3. Die scheinbar ereignislose Induktionszeit kann auch meßtechnisch begründet werden. Jedes Nachweisgerät besitzt eine endliche Empfindlichkeit und kann deshalb erst nach Überschreitung eines durch die Ansprechempfindlichkeit bestimmten Schwellwertes reagieren. Es erhebt sich die Frage, ob die Induktionszeit nicht ebenso als relatives kristallisationskinetisches Maß benutzt werden, wie die häufig benutzte Halbwertszeit der Kristallisation.

Voraussetzung fuir die Anwendung der Induktionszeit als kristallisationskinetisches Maß ist, daß dieser Schwellwert der Kristallinität unabhängig von der Probe gilt. Nur dann kann auch ohne Kenntnis des konkreten Schwellwerts der Kristallinität die Kristallisationskinetik vergleichend beschrieben werden. Vorsicht ist allerdings beim Vergleich von Proben geboten, deren Wachstumsmechanismen sich deutlich voneinander unterscheiden.
Bei realen Kristallisationsprozessen wird der Bruttoeffekt durch die beiden Elementarprozesse der Keimbildung und des Kristallwachstums bestimmt. Um deren Einfluisse zu verdeutlichen, untersuchen wir die Avrami-Gleichung. Für sehr kleine Zeiten läßt diese sich in guter Näherung durch das erste Glied einer Reihe ersetzen:

$$
1-X(t)=\exp \left\{k t^{n}\right\} \approx 1-k t^{n}
$$

Dabei bedeuten $\mathrm{X}(\mathrm{t})$ die Kristallinität, $\mathrm{k}$ die Geschwindigkeitskonstante, $\mathbf{n}$ den Dimensionsfaktor, $\mathbf{t}$ die Zeit. Die Gleichung beschreibt in der genäherten Form das ungestörte Wachstum, bevor die Wachstumsfronten aufeinandertreffen. Unter der Annahme, daß zur Induktionszeit immer der gleiche Kristallisationsgrad erreicht ist, läßt sich der Zusammenhang der Induktionszeit mit der Wachstumsgeschwindigkeit und der Keimbildung bestimmen:

Gelte bei $\mathbf{t}=\mathbf{t}_{\text {ind }}$ für den kristallisierten Anteil $\mathbf{X}(\mathbf{t})=\mathbf{X}\left(\mathbf{t}_{\text {ind }}\right)=$ const, so ergibt sich

$\mathbf{t}_{\text {ind }}=\left(\frac{X\left(t_{\text {ind }}\right)}{k}\right)^{\frac{1}{n}}$

Für den bei handelsuiblichen Kunststoffgranulaten meistens vorliegenden Fall der athermischen Keimbildung gilt bei kugelförmig wachsenden Sphärolithen

$\mathrm{n}=3$ sowie $\mathrm{k}=\frac{4 \pi}{3} \dot{\mathrm{G}}^{3} \mathrm{~N}$,

wobei $\dot{\mathbf{G}}$ die lineare Wachstumsgeschwindigkeit der Sphärolithe, N die Keimzahl pro Volumeneinheit bedeuten. Aus der Näherungsbeziehung für $\mathbf{t}_{\text {ind }}$ ergibt sich unter Beachtung der Konstanz von $\mathbf{X}(\mathrm{t})$ und der plausiblen Annahme einer nur vom Ausgangsmaterial abhängigen und damit konstant zu haltenden Keimdichte $\mathbf{N}$ : Es ist also gerechtfertigt, die reziproke Induktionszeit

$\mathbf{t}_{\text {ind }} \approx \frac{1}{\dot{\mathrm{G}}}$

in dem diskutierten Fall als relatives Maß der Kristallisationsgeschwindigkeit zu nutzen.

Im Falle athermischer Keimbildung und stäbchenförmigem Wachstum mit dem charakteristischen Stäbchendurchmesser $\mathbf{d}$ gilt $\mathbf{n}=\mathbf{2}$ und $\mathbf{k}=\pi \dot{\mathbf{G}}^{2} \mathbf{N d}$, was wieder unter der Voraussetzung einer allein veränderlichen Wachstumsgeschwindigkeit zum gleichen Ergebnis fuihrt.

Abschließend muß noch darauf hingewiesen werden, daß in den Fällen thermischer (homogener) Keimbildung die Zusammenhänge komplizierter werden.

Zusammenfassend kann festgestellt werden, daß bei athermischer Keimbildung und anschließendem Wachstum in ein, zwei oder drei Dimensionen die indirekte Proportionalität zwischen der Induktionszeit und der linearen Wachstumsgeschwindigkeit gilt. Die Keimzahl wird dabei als konstant angesehen und in die Proportionalitätskonstante einbezogen.

Setzt man thermische Keimbildung voraus und benutzt die isokinetische Näherung [6], [12], die besagt, daß 
sowohl Keimbildung als auch Kristallwachstum der gleichen Temperaturabhängigkeit genügen, dann kann auch für diesen Fall eine indirekte Proportionalität zwischen Induktionszeit und Keimbildung/Kristallwachstum abgeleitet werden.

Denkt man sich den realen Keimbildungsvorgang als eine Kombination aus thermischer und athermischer Komponente, so bleibt der prinzipielle, qualitative $\mathrm{Zu}$ sammenhang zwischen Induktionszeit und bestehen, wenn die Größe der beiden Komponenten unabhängig von der Temperatur ist.

\section{Literatur}

[1] Stein, R. S.: Polym. Eng. Sci. 16 (1976) 152

[2] Yoon, M. G., Murakami, S., Katayama, K.: J. Korean Soc. of Text. Eng. and Chem. 20 (1983) 1

[3] Wunderlich, B., Arakawa, T.: J. Polym. Sci. 2 (1964) 3697

[4] Nogami, K., Murakami, S., Katayama, K., Kobayashi, K.: Bull. Inst. Chem. Res. Kyoto Univ. 55 (1977) 227

[5] Ehrenstein, G. W., Maertin, Cl., Pornnimit, B., Scherz, D.: PLASTverarbeiter 37 (1986) Nr. 10

[6] Ziabicki, A.: Colloid \& Polym. Sci. 252 (1974) 207

[7] Barham, P. J., Keller, A.: J. Mater. Sci. 11 (1976) 27

[8] Ehrenstein, G. W., Maertin, Cl.: Kunststoffe 75 (1985) 105

[9] Keller, A.: J. Polym. Sci., Polym. Symp. 58 (1977) 395

[10] Turnbull, D., Fisher, J.C.: J. Chem. Phys. 17 (1949) 71

[11] Frenkel, S. Ja., Baranov, V. G., Eljasevic, G. K.: Acta Polymerica 35 (1984) 393

[12] Nakamura, K., Watanabe, K., Katayama, K., Amano, T.: J. Appl. Polym. Sci. 16 (1972) 1077

[13] Keller, A., Machin, M. J.: J. Macromol. Sci. (Phys.) B1 (1967) 41

[14] Hoffman, J. D.: J. Res. Nat. Bur. Stand (U.S.) 359

[15] Pennings, A. J., Kiel, A. M.: Kolloid-Z, Z. Polym. 205 (1965) 160

[16] Marichin, V. A., Mjasnikova, L. P., Zenke, D., Hirte, R., Weigel, P.: Vysokomol. Soed. Serija B: Kratkie Soobc. 24 (1984) 210; Polymer Bulletin (USA) 12 (1984) 287

\section{Verfasser}

Prof. Dr. rer. nat. habil. Rolf Hirte Dipl.-Phys. Jörg-Peter Melior Technische Fachhochschule Wildau Fachbereich Verfahrenstechnik Tel. (0 33 75) 507-140 